\title{
Thalamocortical functional connectivity in patients with insomnia using resting-state fMRI
}

\author{
Nambeom Kim*, PhD; Eunsoo Won*, MD, PhD; Seo-Eun Cho, MD; \\ Chang-Ki Kang, PhD; Seung-Gul Kang, MD, PhD
}

\begin{abstract}
Background: Insomnia is a common disorder that affects a vast number of patients; the hyperarousal theory of insomnia postulates that patients with insomnia are physiologically activated not only at nighttime but also during the daytime. We aimed to investigate the differences in the resting-state functional connectivity (RSFC) of the thalamus with cortical areas between patients with insomnia disorder and healthy controls. Methods: All participants completed clinical questionnaires and underwent portable polysomnography and resting-state fMRI. Results: Patients in the insomnia group $(n=50)$ showed increased RSFC between the thalamus and right medial superior frontal area, bilateral middle temporal areas, left rectus and right parahippocampal areas compared with controls $(n=42)$ after controlling for age, sex and education level. Among the pairs that showed increased connectivity, several functional connections were negatively correlated with sleep efficiency, measured by polysomnography. Limitations: We used a small sample size. Conclusion: We consider these results on increased thalamocortical hyperactivity in brain areas related to sensory functions as providing evidence for the hyperarousal theory of insomnia.
\end{abstract}

\section{Introduction}

Insomnia is one of the most prevalent and functionally impairing sleep disorders. Approximately one-third of adults experience insomnia symptoms on a weekly basis ${ }^{1,2}$ and $10 \%-20 \%$ of the population suffer from chronic insomnia. ${ }^{3}$ Insomnia is related to impairment of quality of life and functional impairment, as well as mental and physical diseases. ${ }^{4}$ Hyperarousal has long been considered an important mechanism underlying the deprivation of nighttime sleep quality in patients with insomnia. Patients with insomnia show increased nocturnal physiologic activation, such as increased heart rate and basal skin resistance, compared with healthy controls. ${ }^{5}$ Patients with sleep-onset insomnia exhibit increased electromyography discharges of the frontalis and mentalis, and increased $\beta$ frequencies in their electroencephalogram before sleep onset. ${ }^{6}$ Although patients with insomnia often report daytime fatigue, they find it difficult to nap during the daytime, in addition to not being able to sleep during the nighttime. ${ }^{7}$ Patients also exhibit daytime hyperarousal symptoms, including alertness, emotional arousal and physiologic activation. ${ }^{8,9}$ Previous studies on molecular imaging with positron emission tomography showed evidence of hyperarousal in patients with insomnia during the daytime. ${ }^{10}$

To investigate the neurobiological mechanism of this hyperarousal theory of insomnia, several resting-state functional MRI (rs-fMRI) studies have been conducted. ${ }^{11-14}$ A 2013 study based on the hyperarousal theory that included 58 healthy controls of both sexes reported subjective difficulty in falling asleep to be associated with greater resting-state functional connectivity (RSFC) between primary sensory and supplementary motor regions. ${ }^{12}$ A 2014 study involving 15 healthy controls and 15 primary patients with insomnia reported that the RSFC between the bilateral superior parietal lobe and the right ventral anterior cingulate cortex, left ventral posterior cingulate cortex, right splenium of the corpus callosum, right inferior frontal gyrus and right insular lobe was stronger in the primary insomnia group than in the control group. ${ }^{11}$ Another 2014 study involving $17 \mathrm{fe}-$ male patients with insomnia and 17 female healthy controls reported that the patients with insomnia had greater involvement of the anterior insula with the salience networks than the control group did. ${ }^{14}$ However, a 2018 quantitative coordinate-based meta-analysis that included 19 structural and functional neuroimaging studies for patients with insomnia disorder failed to

Correspondence to: S.-G. Kang, Department of Psychiatry, Gil Medical Center, Gachon University College of Medicine, Incheon, Republic of Korea; kangsg@gachon.ac.kr

*These authors contributed equally to this work.

Submitted April 29, 2021; Revised July 21, 2021; Accepted September 6, 2021

Cite as: J Psychiatry Neurosci 2021 November 23;46(6). doi: 10.1503/jpn.210066 
identify consistent patterns of abnormal brain alterations in insomnia disorder. ${ }^{15}$

The thalamus is a brain area composed of grey matter that projects to the cerebral cortex in all directions and functions as an integrative hub for the exchange of information. ${ }^{16}$ It has several functions, such as relaying sensory and motor signals to the cerebral cortex, and regulating sleep, alertness and consciousness. ${ }^{16}$ The thalamus plays a critical role in various aspects of sleep physiology, ${ }^{17}$ such as the generation of sleep spindles ${ }^{18}$ and the propagation of K-complexes. ${ }^{19}$ The transition from wakefulness to sleep is modulated by thalamocortical functional connectivity, ${ }^{17}$ and a decrease in sleep-related compared with wakefulness thalamocortical functional connectivity with extensive cortical regions has been reported..$^{20,21}$ However, a 2016 study reported that an increase in thalamocortical functional connectivity with sensorimotor cortices occurred during the advancement of sleep, from wakefulness to stage 2 sleep, which was attributed to the effects of K-complexes and sleep spindles. ${ }^{17}$ Although previous studies have been conducted on functional connectivity between the thalamus and other cortical areas during the sleep initiation period, ${ }^{8}$ there are a limited number of studies that have compared the RSFC between the thalamus and other brain areas of patients with insomnia and healthy controls during the daytime. Furthermore, we are unaware of any investigations of the correlations between functional connectivity measures of brain areas that show substantial differences in RSFC and objective polysomnographic sleep measurements.

We hypothesized that RSFC between the thalamus and several cortical areas will increase during the daytime in the insomnia group, representing hyperarousal of brain areas associated with sleep physiology in patients with insomnia. Furthermore, we hypothesized that significant negative correlations would exist between RSFC of these brain areas and sleep efficiency in the insomnia group, which would indicate that the hyperarousal state of brain areas associated with sleep physiology negatively affect sleep quality in patients with insomnia. Our aims were to compare the differences in RSFC between the thalamus and cortical areas of patients with insomnia and healthy controls, and to investigate the correlation between RSFC and sleep efficiency measured by polysomnography (PSG) in the insomnia group.

\section{Methods}

\section{Participants and clinical assessment}

All participants were recruited from the Sleep Medicine Center of the Psychiatric Department at the Gil Medical Center, Gachon University College of Medicine, Incheon, Republic of Korea. Our inclusion criteria for patients with insomnia disorder were as follows: age 18-70 years and right-handed, met the diagnostic criteria of insomnia disorder found in the Diagnostic and Statistical Manual of Mental Disorders, fifth edition (DSM-5), ${ }^{22}$ duration of insomnia at least 3 times a week for 6 months or longer, a score of 8 or more on the Korean version of the Pittsburgh Sleep Quality Index (PSQI) at screening, ${ }^{23}$ no use of any psychiatric medications including hypnotics in the previous 14 days and no cognitive behavioural therapy for insomnia in the previous 14 days. Our inclusion criteria for good sleepers were as follows: age 18-70 years and right-handed, no symptoms or history of sleep disorders including insomnia, a PSQI score of 4 or less at screening and no history of use of sleep or psychiatric medications.

Our common exclusion criteria for both groups were as follows: current diagnosis of moderate-to-severe sleep disorders such as sleep apnea, restless legs syndrome, circadian rhythm sleep disorders, parasomnia and narcolepsy other than insomnia disorder determined by history and screening scales; shift worker or traveller having frequent jet lag; high risk of sleep apnea according to the Korean Berlin Sleep Questionnaire, ${ }^{24}$ obesity with a body mass index over 30; evidence in PSG of a sleep disorder other than insomnia; current or a history of other comorbid psychiatric disorders (except insomnia disorder) including major depressive disorders; bipolar disorders; psychotic disorders; alcohol, caffeine or substance use disorders; personality disorders; major neurocognitive disorders or intellectual disability based on a clinical interview; presence or a history of substantial medical or neurologic diseases, such as epilepsy, cerebrovascular disease or Parkinson disease; contraindications for 3T MRI, such as metal in the body or claustrophobia; pregnancy or lactation; and abnormal finding on structural brain MRI.

All participants were screened through phone interviews, and after the initial screening, board-certified psychiatrists with a specialty in sleep medicine evaluated the eligibility of each participant via a face-to-face interview to assess whether the participant met the diagnostic criteria for insomnia disorder, administer a semistructured interview for psychiatric and sleep disorders, and compile the medical history of each participant. We obtained written informed consent from all participants before inclusion in the study, and the Institutional Review Board of Gil Medical Center approved all study protocols.

All participants were instructed to complete a questionnaire on their demographic characteristics, sleep status, and consumption of caffeine, alcohol, and nicotine. The participants also completed the Korean version of the PSQI $^{23}$ and the Berlin Questionnaire ${ }^{24}$ during screening. They also completed the Korean versions of the PSQI and Insomnia Severity Index (ISI) $)^{25}$ on the day of MRI scanning.

\section{Polysomnography to exclude sleep disorders other than insomnia}

To exclude participants with occult sleep disorders other than insomnia, an overnight PSG was performed using the Embletta MPR sleep system (Natus). The participants were instructed to go to sleep at the same time as usual on PSG days. Sleep scoring was visually performed by an experienced sleep technician and a sleep specialist medical doctor (S.G.K.) according to the American Academy of Sleep Medicine scoring manual (version 2.4.), ${ }^{26}$ with RemLogic 3.4.4 (Embla Systems) as the scoring platform. We excluded participants with an apnea-hypopnea index (AHI) of 15 or more, 15 or more periodic limb movements during sleep, or evidence of rapid eye movement sleep behaviour disorder in the PSG. 


\section{Functional MRI acquisition}

Functional MRI was conducted between 9:30 am and 1:00 pm, at least 2 hours after waking and before lunch, and within 2 weeks after the PSG. We used a $3 \mathrm{~T}$ whole-body scanner (TrioTim Syngo; Siemens) for functional image acquisition with an interleaved $T_{2}{ }^{*}$-weighted EPI gradient echo sequence (repetition time [TR] $2500 \mathrm{~ms}$, echo time [TE] $25 \mathrm{~ms}$, flip angle $90^{\circ}$, slice thickness $3.5 \mathrm{~mm}$, in-plane resolution $3.5 \mathrm{~mm} \times 3.5 \mathrm{~mm}$ and matrix size $64 \times 64$ ) with a 12-channel birdcage head coil. A total of 240 functional volumes were acquired for each participant. After rs-fMRI acquisition, an anatomic image was acquired using a high, $T_{1}$-weighted, 3D gradient echo pulse sequence with magnetization prepared rapid gradient echo (TR $1900 \mathrm{~ms}$, TE $3.3 \mathrm{~ms}$, inversion time [TI] $900 \mathrm{~ms}$, flip angle $9^{\circ}$, slice thickness $1.0 \mathrm{~mm}$, in-plane resolution $0.5 \mathrm{~mm} \times 0.5 \mathrm{~mm}$, field of view $250 \mathrm{~mm}$ and matrix size $416 \times 512){ }^{27}$

\section{Image processing and analysis of fMRI data}

We used SPM12 (Wellcome Trust Centre for Neuroimaging, London, UK) to reprocess the rs-fMRI data. First, the slice timing difference was corrected and the centre of the image was relocated near the anterior commissure. Then, rs-fMRI and $T_{1}$-weighted images were imported to the CONN FC toolbox v19c (http://www.nitrc.org/projects/conn) for further preprocessing. To correct for the between-scan rigid body motion, we realigned the functional images to the first image in the time series. The anatomic image was co-registered based on the mean functional image, and all functional images were spatially normalized to the Montreal Neurological Institute space using a transformation matrix derived from the $T_{1}$-weighted anatomic image segmentation. The functional images were then resliced to $2 \mathrm{~mm} \times 2 \mathrm{~mm} \times$ $2 \mathrm{~mm}$ and spatially smoothed using a Gaussian kernel with $8 \mathrm{~mm}$ full-width at half maximum. We also modelled 6 motion parameters obtained from the realignment as nuisance covariates. We performed seed-to-voxel analysis in the right and left thalamic regions as seed regions. The right and left thalamic regions were predefined using the Harvard-Oxford atlas (FMRIB Software Library, Oxford, UK).

\section{Statistical analysis}

\section{Clinical data}

SPSS Statistics for Windows (version 25.0, IBM Corp.) was used to analyze the clinical data. We used $\chi^{2}$ or Fisher exact tests (for categorical variables) and independent $t$ tests to compare demographic and clinical characteristics between groups. We set statistical significance as a $p$ value of less than 0.05 .

\section{Correlational analyses}

We conducted group-level analysis between the insomnia and control groups using a general linear model with age, sex and education level as covariates. We set the statistical thresholds to a voxel-wise uncorrected $p$ value of less than 0.001 and a cluster-wise FDR corrected $p$ value of 0.05 .
To explore the associations between sleep efficiency measured by PSG and functional connectivity, we extracted the zscores of the connectivity of cortical regions to the seed from all the clusters showing statistical significance in the seed-tovoxel analysis. To calculate the partial correlation while controlling for the effect of nuisance covariates (age, sex and education level), we performed least-squares regression twice: between clinical data and nuisance covariates, and between mean Fisher z-scores and nuisance covariates. In each regression analysis, we estimated the residuals of clinical data and of mean Fisher z-scores. Partial correlation coefficients were calculated between the residual of sleep efficiency and the residual of mean Fisher z-scores of the connected cortical regions. Partial correlation analysis was performed only in the insomnia group to exclude group effects.

\section{Results}

\section{Demographic and clinical characteristics of participants}

We recruited a total of 192 participants (101 patients with insomnia and 91 controls) who passed the initial telephone screening for the study. We excluded 68 patients with insomnia and 61 controls based on the following criteria: not appropriate for the study based on the screening scale (patients with insomnia $n=36$, controls $n=30$ ), deemed ineligible after face-to-face interview (patients with insomnia $n=8$, controls $n=5$ ), metal in the body (patients with insomnia $n=$ 7 , controls $n=8$ ), abnormal polysomnography findings (patients with insomnia $n=3$, controls $n=4$ ), withdrew informed consent or did not attend the clinic on the scanning day (patients with insomnia $n=12$, controls $n=12$ ) or had a newly found structural brain abnormality after MRI (patients with insomnia $n=2$, controls $n=2$ ). After applying the above exclusion criteria, 50 patients with insomnia and 42 healthy controls completed the full research protocol.

The demographic and clinical characteristics of the participants are presented in Table 1 . The age and education level were significantly higher in the insomnia group than in the control group $(p<0.05)$. There were no significant differences between the 2 groups in terms of sex distribution. The average duration of illness in the insomnia-disorder group was 6.9 years. On the day of the MRI scan, the average ISI and PSQI scores differed significantly between the insomnia and control groups. The total sleep time, sleep efficiency and wake-after-sleep onset as measured by PSG were also significantly different between the 2 groups.

\section{Comparison of thalamocortical resting-state functional connectivity between the insomnia and control groups}

Compared with the control group, the insomnia group showed increased RSFC in the right superior medial frontal area, left middle temporal pole, right parahippocampal gyrus, right middle temporal area and left rectus with the right thalamus (Figure 1 and Table 2). The insomnia group also showed increased RSFC in the left superior parietal area, bilateral middle temporal poles and left rectus with the left 
Table 1: Demographic and clinical characteristics of the participants

\begin{tabular}{|c|c|c|c|}
\hline Characteristic & $\begin{array}{c}\text { Insomnia } \\
\text { disorder } \\
n=50\end{array}$ & $\begin{array}{l}\text { Healthy } \\
\text { control } \\
n=42\end{array}$ & Statistics \\
\hline Age, yr & $43.3 \pm 13.9$ & $36.2 \pm 11.2$ & $\begin{aligned} t & =2.71 \\
p & =0.008\end{aligned}$ \\
\hline Sex, female & $33(66.0)$ & $26(61.9)$ & $\begin{array}{c}\chi^{2}=0.17 \\
p=0.7\end{array}$ \\
\hline $\begin{array}{l}\text { Duration of insomnia } \\
\text { disorder, yr }\end{array}$ & $6.9 \pm 7.8$ & - & \\
\hline Duration of education, yr & $13.6 \pm 3.8$ & $15.4 \pm 2.0$ & $\begin{array}{l}t=-2.88 \\
p=0.005\end{array}$ \\
\hline \multicolumn{4}{|l|}{ PSQI* $^{\star}$} \\
\hline Total score & $12.9 \pm 3.6$ & $2.4 \pm 1.3$ & $\begin{array}{l}t=19.25 \\
p<0.001\end{array}$ \\
\hline TST, min & $281.8 \pm 89.0$ & $445.5 \pm 53.8$ & $\begin{array}{c}t=-10.86 \\
p<0.001\end{array}$ \\
\hline SE, \% & $64.9 \pm 20.0$ & $96.2 \pm 4.2$ & $\begin{array}{c}t=-10.79 \\
p<0.001\end{array}$ \\
\hline ISI score ${ }^{\star}$ & $18.5 \pm 4.8$ & $1.3 \pm 1.7$ & $\begin{array}{l}t=23.73 \\
p<0.001\end{array}$ \\
\hline \multicolumn{4}{|l|}{ Polysomnographic data } \\
\hline TST, min & $346.9 \pm 73.3$ & $394.3 \pm 65.4$ & $\begin{array}{l}t=-3.24 \\
p=0.002\end{array}$ \\
\hline SE, \% & $77.7 \pm 12.3$ & $91.1 \pm 7.1$ & $\begin{array}{l}t=-6.51 \\
p<0.001\end{array}$ \\
\hline WASO, min & $73.2 \pm 56.8$ & $31.9 \pm 29.2$ & $\begin{aligned} t & =4.48 \\
p & <0.001\end{aligned}$ \\
\hline $\mathrm{AHI}, \mathrm{no} . / \mathrm{h}$ & $3.4 \pm 3.4$ & $3.0 \pm 4.4$ & $\begin{array}{c}t=0.54 \\
p=0.6\end{array}$ \\
\hline
\end{tabular}

AHI = apnea-hypopnea index; ISI = Insomnia Severity Index; PSQI = Pittsburgh Sleep Quality Index; SE = sleep efficiency; SOL = sleep onset latency; TST = total sleep

time; WASO = wake-after-sleep onset.

Values are reported as mean \pm standard deviation or $n(\%)$

${ }^{\star}$ These scales were completed on the date of the brain MRI scanning.

thalamus compared with the control group (Figure 1 and Table 2). There was no cortical area showing significantly decreased RSFC in the insomnia group compared with the control group. Mean values and standard errors of Fisher zscores for RSFC in the connected cortical regions with the thalamus are shown in Figure 2 and Appendix 1, Table S1, available at jpn.ca.

\section{Correlation between resting-state functional connectivity and sleep efficiency measured by polysomnography in the insomnia group}

Mean Fisher z-scores were extracted from all 9 regions of interest (ROIs; 5 ROIs in relation to the right thalamus and 4 ROIs in relation to the left thalamus) that showed significantly increased RSFC in the insomnia group compared with the healthy control group. We conducted partial correlation analysis in the insomnia group between sleep efficiency measured by PSG and Fisher z-scores of the RSFC from the 9 ROIs after controlling for age, sex and education level. We found 5 areas showing significant correlations $(p<0.05)$. For the right thalamus, these areas were the right superior medial frontal area, left middle temporal pole and right parahippocampal gyrus (Table 3). For the left thalamus, these areas were the bilateral middle temporal poles (Table 3).

\section{Discussion}

We found higher levels of RSFC of the thalamus with several cortical areas during the daytime in the insomnia group compared with the control group, and significant correlations between RSFC and sleep efficiency measured by PSG in the insomnia group. Compared with the control group, the insomnia group showed increased RSFC of the right thalamus with the right medial superior frontal area, bilateral middle temporal area, left rectus and right parahippocampal area, and increased RSFC of the left thalamus with the left superior parietal area, bilateral middle temporal poles and left rectus.

Some of the areas that showed significantly increased RSFC with the thalamus in our study, including the middle temporal, superior parietal and medial superior frontal areas, are regions associated with sensory-related activity. ${ }^{28-30}$ In patients with insomnia, the connectivity between the thalamus and such areas may be increased, which may lead to hypersensitivity to external stimulation and, in turn, lead to a state of hyperarousal. The parahippocampal area is associated with memory encoding and retrieval ${ }^{31}$ and emotional processing. ${ }^{32}$ The gyrus rectus, or straight gyrus, is located at the most medial margin of the inferior surface of the frontal lobe and has been identified as a critical region involved with mood processing. ${ }^{33-38}$ A 2002 study that involved 15 patients with depression and 20 controls reported significantly smaller gyrus rectus cortical volumes compared with controls. ${ }^{34}$ A 2004 study that involved 24 older adults with major depression and 19 group-matched controls reported bilateral volume reductions in grey matter in the gyrus rectus of these patients. ${ }^{33}$ Emotion dysregulation is a common feature in patients with insomnia. ${ }^{39}$ Previous studies have reported increased connectivity of frontal and limbic areas with other brain regions in patients with dysfunctional emotional processing. ${ }^{40,41}$ Our findings of increased RSFC between the thalamus and parahippocampal area and left rectus may represent emotional dysregulation in insomnia patients, which may lead to a state of hyperarousal that contributes to insomnia symptoms. ${ }^{42} \mathrm{~A}$ study that involved 13 patients with insomnia and 18 healthy controls reported that RSFC between the thalamus and the prefrontal cortex was increased in the insomnia group compared with the healthy control group, and RSFC between the thalamus and the parietal cortex in the insomnia group decreased after cognitive behavioural therapy for insomnia (CBT-I). ${ }^{43}$ The authors of the study considered their results as providing a neural basis for sensory-related hyperarousal in insomnia, based on the observed hyperactivity of the thalamus in relation to cortical excitability. The authors also suggested that the basis for the treatment effects of CBT-I on hyperarousal symptoms was the decrease in thalamus activity in relation to the parietal cortex, which coincides with our findings. However, a study that involved 27 patients with insomnia and 39 healthy controls, which had investigated RSFC between the thalamus and other brain areas during waking periods, reported decreased RSFC of the thalamus with the orbitofrontal cortex, anterior cingulate cortex, hippocampus, putamen and caudate in the patients with insomnia compared with the controls. ${ }^{44}$ The authors associated the decrease in 


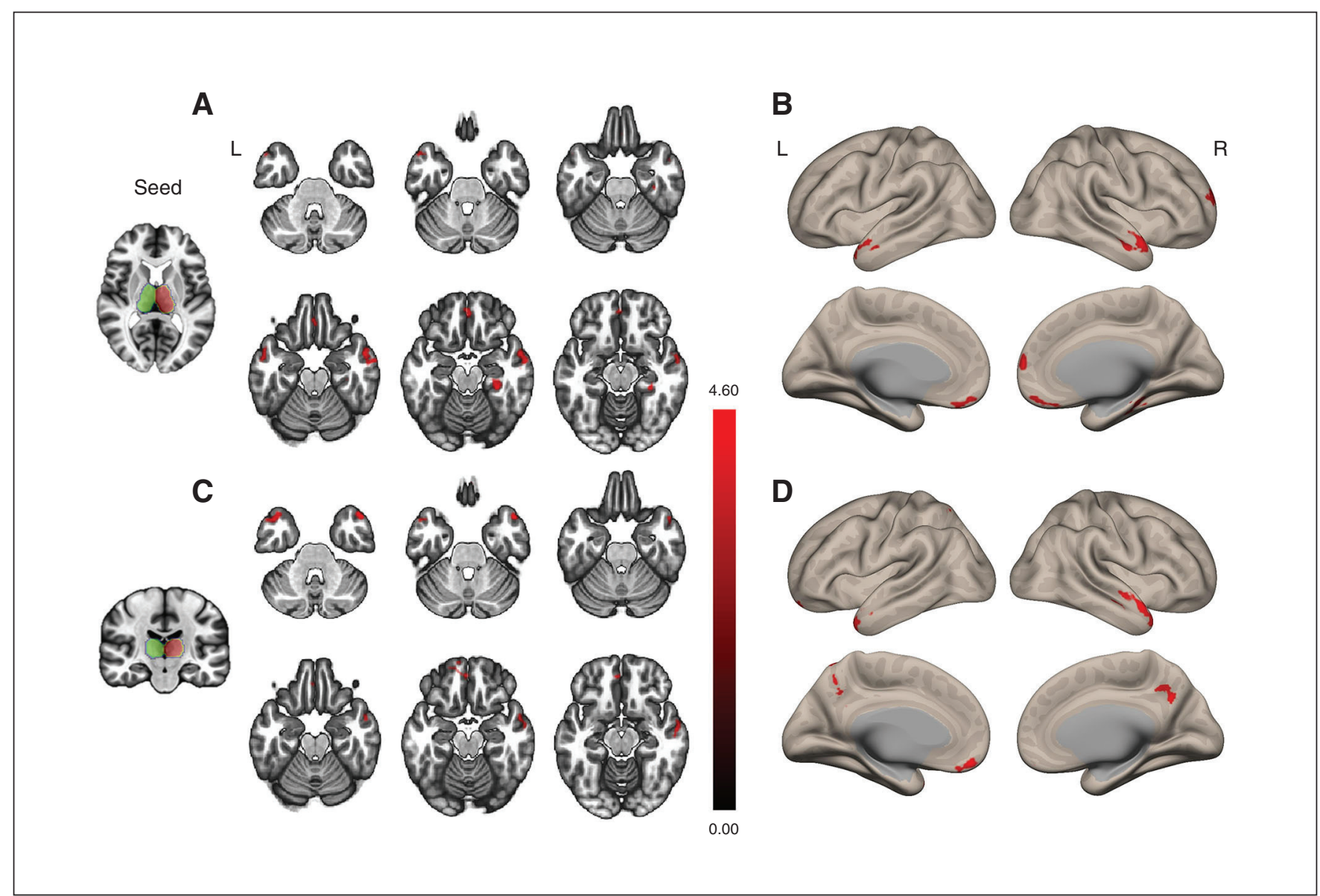

Figure 1: Cortical areas of increased thalamocortical functional connectivity in participants with insomnia compared with healthy controls. (A and B) The seed is the right thalamus. The insomnia group showed increased functional connectivity in the right superior medial frontal area, bilateral middle temporal areas, right parahippocampal gyrus and left rectus (areas in red) with the right thalamus compared with the healthy control group. ( $C$ and $D$ ) The seed is the left thalamus. The insomnia group showed increased functional connectivity in the left superior parietal area, both mid-temporal poles and left rectus (areas in red) with the left thalamus compared with the healthy control group. The green and red contours represent the left and right seeds of the thalamus, respectively. The statistical threshold was a voxel-wise uncorrected $p<0.001$, with a cluster-wise false discovery rate corrected $p<0.05$.

\begin{tabular}{|c|c|c|c|c|c|c|}
\hline \multirow{2}{*}{$\begin{array}{l}\text { Seed } \\
\text { Right thalamus }\end{array}$} & \multirow{2}{*}{$\begin{array}{l}\text { Connected cortical region } \\
\text { Frontal_Sup_Medial_R }\end{array}$} & \multicolumn{3}{|c|}{$\begin{array}{l}\text { MNI coordinates } \\
\quad(x, y, z)\end{array}$} & \multirow{2}{*}{$\begin{array}{c}\begin{array}{c}\text { Cluster } \\
\text { size }\end{array} \\
155\end{array}$} & \multirow{2}{*}{$\begin{array}{c}\text { z-score } \\
3.98\end{array}$} \\
\hline & & 8 & 58 & 12 & & \\
\hline & Temporal_Pole_Mid_L & -54 & 12 & -34 & 145 & 3.91 \\
\hline & ParaHippocampal_R & 30 & -32 & -16 & 156 & 3.91 \\
\hline & Temporal_Mid_R & 66 & -4 & -20 & 287 & 3.83 \\
\hline & Rectus_L & -2 & 48 & -16 & 154 & 3.77 \\
\hline \multirow[t]{4}{*}{ Left thalamus } & Parietal_Sup_L & -18 & -60 & 52 & 344 & 4.38 \\
\hline & Temporal_Pole_Mid_R & 48 & 16 & -32 & 337 & 4.27 \\
\hline & Rectus_L & -4 & 48 & -16 & 159 & 3.97 \\
\hline & Temporal_Pole_Mid_L & -44 & 12 & -34 & 189 & 3.93 \\
\hline
\end{tabular}

Frontal_Sup_Medial_R = right medial superior frontal area; MNI = Montreal Neurological Institute;

ParaHippocampal_R = right parahippocampal area; Parietal_Sup_L = left superior parietal area; Rectus_L = left rectus;

Temporal_Mid_R = right middle temporal area; Temporal_Pole_Mid_L = left middle temporal pole; Temporal_Pole_

Mid $R=$ right middle temporal pole.

We performed this analysis after controlling for age, sex and education level. 
RSFC between the thalamus and orbitofrontal cortex with a decrease in decision-making and goal-directed behaviour in the patients with insomnia, and a reduction in RSFC between the thalamus and anterior cingulate cortex with dysfunctional emotional processing in these patients. Although the gyrus rectus is in anatomic proximity to the orbitofrontal cortex and anterior cingulate cortex, previous studies have distinguished the individual functions of these regions. ${ }^{33,45}$ Therefore, we

A

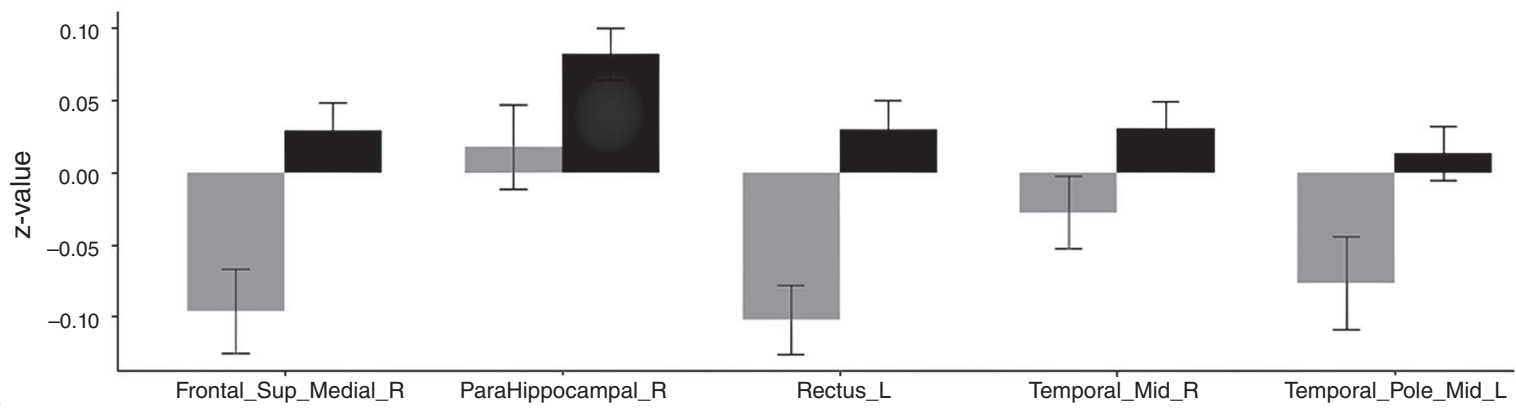

B

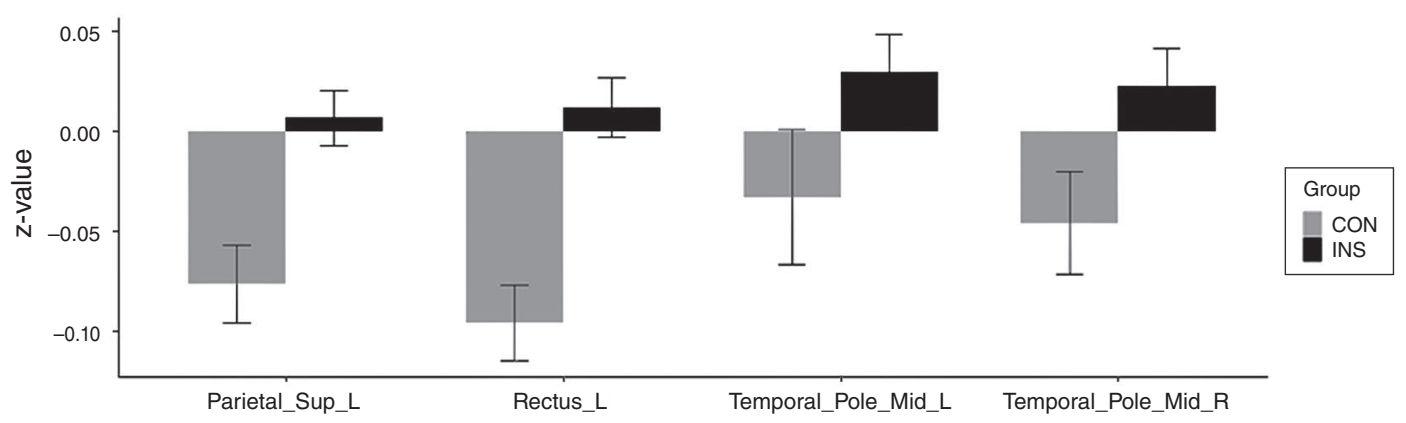

Figure 2: Mean and standard errors for Fisher z-scores of resting-state functional connectivity between the thalamus and cortical regions. Resting-state functional connectivity was defined as Fisher z-scores in the INS and CON groups. (A) Correlations between the right thalamus and cortical regions and $(\mathrm{B})$ between the left thalamus and cortical regions. Bars and whiskers represent means and standard errors. CON $=$ healthy control; Frontal_Sup_Medial_R = right medial superior frontal area; INS = insomnia; ParaHippocampal_R = right parahippocampal area; Parietal_Sup_L = left superior parietal area; Rectus_L = left rectus; Temporal_Mid_R = right middle temporal area; Temporal_Pole_ Mid_L = left middle temporal pole; Temporal_Pole_Mid_R = right middle temporal pole.

\begin{tabular}{|c|c|c|c|c|}
\hline Clinical index & Seed & Connected cortical region & Pearson coefficient & $p$ value \\
\hline \multirow{9}{*}{$\begin{array}{l}\text { Sleep efficiency from } \\
\text { PSG }\end{array}$} & \multirow[t]{5}{*}{ Right thalamus } & Frontal_Sup_Medial_R & -0.28 & 0.049 \\
\hline & & Temporal_Pole_Mid_L & -0.284 & 0.045 \\
\hline & & ParaHippocampal_R & -0.295 & 0.04 \\
\hline & & Temporal_Mid_R & -0.219 & 0.1 \\
\hline & & Rectus_L & -0.181 & 0.2 \\
\hline & \multirow[t]{4}{*}{ Left thalamus } & Parietal_Sup_L & 0.036 & 0.8 \\
\hline & & Temporal_Pole_Mid_R & -0.364 & 0.009 \\
\hline & & Rectus_L & -0.113 & 0.4 \\
\hline & & Temporal_Pole_Mid_L & -0.229 & 0.035 \\
\hline
\end{tabular}

Frontal Sup Medial $R=$ right medial superior frontal area; ParaHippocampal_ $R=$ right parahippocampal area; Parietal Sup_L = left superior parietal area; PSG = polysomnography; Rectus_L = left rectus; Temporal_Mid_R = right middle temporal area; Temporal_Pole_Mid_L = left middle temporal pole; Temporal_Pole_Mid_R = right middle temporal pole.

We performed the partial correlation analysis between sleep efficiency from PSG and thalamocortical connectivity after controlling for age, sex and educational level. 
considered the differences between our findings and those of previous studies to represent functional differences of specific brain areas related to sleep physiology.

Other studies have investigated RSFC of the whole brain or used brain regions other than the thalamus as a seed, such as the amygdala or insula, and have reported significant differences in RSFC between such seed regions and various brain areas, including the thalamus. A 2017 study that involved 57 patients and 46 healthy controls reported that patients with insomnia were found to have enhanced RSFC between the left insula and bilateral thalamus, right ACC and left precuneus. ${ }^{46}$ A 2017 randomized controlled consort trial that involved 27 healthy participants with insomnia symptoms and 27 healthy participants without insomnia symptoms compared the voxel-mirrored homotopic connectivity (VMHC) of the 2 groups, which represents interhemispheric coordination, and reported that the participants with insomnia symptoms displayed increased VMHC in the bilateral thalamus/posterior insula, inferior parietal lobe, fusiform, middle cingulate gyrus and postcentral gyrus. ${ }^{47}$ Increased RSFC between the amygdala and the premotor and sensorimotor cortex, and decreased RSFC between the amygdala and the thalamus, insula and striatum in patients with insomnia were reported in a 2012 study that involved 10 patients with insomnia and 10 healthy controls; the authors compared RSFC between the amygdala and other wholebrain regions. ${ }^{13}$ In a 2016 study that used rs-fMRI to evaluate the difference in alterations of spontaneous brain activity between healthy participants with and without insomnia symptoms, ${ }^{48}$ participants with insomnia symptoms showed decreased fractional amplitude of low-frequency fluctuation (fALFF) in the left thalamus, left ventral anterior insula, bilateral posterior insula and pons. The authors stated that their finding of altered fALFF in the left thalamus supported the hyperarousal theory of insomnia symptoms.

Although we could not separately investigate RSFC of the thalamic reticular nucleus with the thalamus because of the characteristics of its anatomic structure, we consider the thalamic reticular nucleus is important with respect to thalamic function and sleep physiology. The thalamic reticular nucleus is considered to be an important area in generating sleep patterns, along with the thalamus. ${ }^{49}$ It has been suggested that the reticular nucleus regulates the flow of information from the thalamic nuclei to the cortex.$^{50}$ GABAergic neurons of the thalamic reticular nucleus are inhibitory to the thalamic nuclei and thalamocortical neurons. ${ }^{51}$ When the regulating activity of the thalamic reticular nucleus over the thalamus is altered, the connectivity of the thalamus to other cortical regions may in turn be affected.

In our partial correlation analysis, as sleep efficiency measured by PSG decreased, RSFC between the right thalamus and right medial superior frontal area, left middle temporal pole and right parahippocampal gyrus, and RSFC between the left thalamus and both middle temporal poles increased in the insomnia group. The RSFC of brain areas that showed significant differences between groups also showed significant negative correlations with sleep efficiency measured by PSG, which suggests an association between objective insomnia symptoms and increases in RSFC. A previous study reported correlations between subjective rating scales such as the PSQI and ISI, and sleep efficiency from sleep diaries, with RSFC of specific brain areas. ${ }^{43}$ Our findings suggest significant correlations between objective sleep measurements and RSFC.

\section{Limitations}

We relied on a small sample size. Although our sample size was similar to those of recent rs-fMRI studies, future studies with a larger sample size may provide more confident results. Our findings showed that age and education level were significantly higher in the insomnia group than in the control group. Therefore, we conducted a group-level analysis between the insomnia and control groups using a general linear model with age, sex and education level as covariates. In addition, we conducted a correlation analysis between sleep efficiency and functional connectivity while controlling for the effect of nuisance covariates, including age, sex and education level. However, adding these variables as nuisance variables may not fully correct for the mismatch in age and education between the 2 groups, and this may have confounded our results.

\section{Conclusion}

Our fMRI findings suggest increased connectivity of the thalamus with several other brain regions in patients with insomnia relative to controls during the resting state of the waking period. We consider our results as neurobiological evidence supporting the hyperarousal theory of insomnia.

Affiliations: From the Department of Biomedical Engineering Research Center (N. Kim); Department of Psychiatry (S.-G. Kang, S.-E. Cho), Gil Medical Center, College of Medicine; and Department of Radiological Science (C.-K. Kang), College of Health Science, Gachon University, Incheon, Republic of Korea; Department of Psychiatry, CHA Bundang Medical Center, CHA University, Seongnam, Republic of Korea (E. Won); Department of Psychiatry, Chaum, Seoul, Republic of Korea (E. Won).

Funding: This work was supported by the National Research Foundation of Korea (NRF), a grant funded by the Korean government (MSIT) (grant no. NRF-2020R1A2C1007527). This research was also supported by the Basic Science Research Program through the NRF, funded by the Ministry of Education (NRF-2017R1D1A1B03032431).

\section{Competing interests: None declared.}

Contributors: Seung-Gul Kang designed the study. Seung-Gul Kang and Seo-Eun Cho acquired the data, which Nambeom Kim and Chang-Ki Kang analyzed. Seung-Gul Kang, Eunsoo Won and Nambeom Kim wrote the article, which was reviewed by all of the authors. All of the authors gave final approval of the version to be published and agreed to be accountable for all aspects of the work.

Content licence: This is an Open Access article distributed in accordance with the terms of the Creative Commons Attribution (CC BY-NC-ND 4.0) licence, which permits use, distribution and reproduction in any medium, provided that the original publication is properly cited, the use is noncommercial (i.e., research or educational use), and no modifications or adaptations are made. See: https://creativecommons.org/licenses/by-nc-nd/4.0/ 


\section{References}

1. Winkelman JW. CLINICAL PRACTICE. Insomnia disorder. N Engl J Med 2015;373:1437-44.

2. Ohayon MM. Epidemiology of insomnia: what we know and what we still need to learn. Sleep Med Rev 2002;6:97-111.

3. Chung KF, Yeung WF, Ho FY, et al. Cross-cultural and comparative epidemiology of insomnia: the Diagnostic and Statistical Manual (DSM), International Classification of Diseases (ICD) and International Classification of Sleep Disorders (ICSD). Sleep Med 2015;16:477-82.

4. Blank M, Zhang J, Lamers F, et al. Health correlates of insomnia symptoms and comorbid mental disorders in a nationally representative sample of US adolescents. Sleep (Basel) 2015;38:197-204.

5. Monroe LJ. Psychological and physiological differences between good and poor sleepers. J Abnorm Psychol 1967;72:255-64.

6. Freedman RR, Sattler HL. Physiological and psychological factors in sleep-onset insomnia. J Abnorm Psychol 1982;91:380-9.

7. Bonnet MH, Arand DL. Hyperarousal and insomnia. Sleep Med Rev 1997;1:97-108.

8. Regestein QR, Dambrosia J, Hallett M, et al. Daytime alertness in patients with primary insomnia. Am J Psychiatry 1993;150:1529-34.

9. Soldatos CR, Kales A. Sleep disorders: research in psychopathology and its practical implications. Acta Psychiatr Scand 1982;65:381-7.

10. Nofzinger EA, Buysse DJ, Germain A, et al. Functional neuroimaging evidence for hyperarousal in insomnia. Am J Psychiatry 2004;161:2126-8.

11. Li Y, Wang E, Zhang H, et al. Functional connectivity changes between parietal and prefrontal cortices in primary insomnia patients: evidence from resting-state fMRI. Eur J Med Res 2014;19:32.

12. Killgore WD, Schwab ZJ, Kipman M, et al. Insomnia-related complaints correlate with functional connectivity between sensorymotor regions. Neuroreport 2013;24:233-40.

13. Huang Z, Liang P, Jia X, et al. Abnormal amygdala connectivity in patients with primary insomnia: evidence from resting state fMRI. Eur J Radiol 2012;81:1288-95.

14. Chen MC, Chang C, Glover GH, et al. Increased insula coactivation with salience networks in insomnia. Biol Psychol 2014;97:1-8.

15. Tahmasian M, Noori K, Samea F, et al. A lack of consistent brain alterations in insomnia disorder: an activation likelihood estimation meta-analysis. Sleep Med Rev 2018;42:111-8.

16. Basso MA, Uhlrich D, Bickford ME. Cortical function: a view from the thalamus. Neuron 2005;45:485-8.

17. Hale JR, White TP, Mayhew SD, et al. Altered thalamocortical and intra-thalamic functional connectivity during light sleep compared with wake. Neuroimage 2016;125:657-67.

18. Contreras D, Destexhe A, Sejnowski TJ, et al. Spatiotemporal patterns of spindle oscillations in cortex and thalamus. J Neurosci 1997;17:1179-96

19. Jahnke K, von Wegner F, Morzelewski A, et al. To wake or not to wake? The two-sided nature of the human K-complex. Neuroimage 2012;59:1631-8.

20. Spoormaker VI, Schroter MS, Gleiser PM, et al. Development of a large-scale functional brain network during human non-rapid eye movement sleep. J Neurosci 2010;30:11379-87.

21. Picchioni D, Pixa ML, Fukunaga M, et al. Decreased connectivity between the thalamus and the neocortex during human nonrapid eye movement sleep. Sleep (Basel) 2014;37:387-97.

22. American Psychiatric Association. Diagnostic and statistical manual of mental disorders (DSM-5). Washington: American Psychiatric Pub; 2013.

23. Sohn SI, Kim DH, Lee MY, et al. The reliability and validity of the Korean version of the Pittsburgh Sleep Quality Index. Sleep Breath 2012;16:803-12.

24. Kang K, Park KS, Kim JE, et al. Usefulness of the Berlin Questionnaire to identify patients at high risk for obstructive sleep apnea: a population-based door-to-door study. Sleep Breath 2013;17:803-10.

25. Cho YW, Song ML, Morin CM. Validation of a Korean version of the Insomnia Severity Index. J Clin Neurol 2014;10:210-5.

26. Berry RB, Brooks R, Gamaldo C, et al. AASM Scoring Manual Updates for 2017 (Version 2.4). J Clin Sleep Med 2017;13:665-6.

27. Kim YB, Kim N, Lee JJ, et al. Brain reactivity using fMRI to insomnia stimuli in insomnia patients with discrepancy between subjective and objective sleep. Sci Rep 2021;11:1592.

28. du Boisgueheneuc F, Levy R, Volle E, et al. Functions of the left superior frontal gyrus in humans: a lesion study. Brain 2006;129:3315-28.
29. Price NS, Born RT. Timescales of sensory- and decision-related activity in the middle temporal and medial superior temporal areas. J Neurosci 2010;30:14036-45.

30. Wolpert DM, Goodbody SJ, Husain M. Maintaining internal representations: the role of the human superior parietal lobe. Nat Neurosci 1998;1:529-33.

31. van Strien NM, Cappaert NL, Witter MP. The anatomy of memory: an interactive overview of the parahippocampal-hippocampal network. Nat Rev Neurosci 2009;10:272-82.

32. Blood AJ, Zatorre RJ, Bermudez P, et al. Emotional responses to pleasant and unpleasant music correlate with activity in paralimbic brain regions. Nat Neurosci 1999;2:382-7.

33. Ballmaier M, Toga AW, Blanton RE, et al. Anterior cingulate, gyrus rectus, and orbitofrontal abnormalities in elderly depressed patients: an MRI-based parcellation of the prefrontal cortex. Am J Psychiatry 2004;161:99-108.

34. Bremner JD, Vythilingam M, Vermetten E, et al. Reduced volume of orbitofrontal cortex in major depression. Biol Psychiatry 2002;51: 273-9.

35. Drevets WC, Price JL, Simpson JR Jr, et al. Subgenual prefrontal cortex abnormalities in mood disorders. Nature 1997;386:824-7.

36. Taylor WD, Steffens DC, McQuoid DR, et al. Smaller orbital frontal cortex volumes associated with functional disability in depressed elders. Biol Psychiatry 2003;53:144-9.

37. Botteron KN, Raichle ME, Drevets WC, et al. Volumetric reduction in left subgenual prefrontal cortex in early onset depression. Biol Psychiatry 2002;51:342-4.

38. MacFall JR, Payne ME, Provenzale JE, et al. Medial orbital frontal lesions in late-onset depression. Biol Psychiatry 2001;49:803-6.

39. Palagini L, Moretto U, Novi M, et al. Lack of resilience is related to stress-related sleep reactivity, hyperarousal, and emotion dysregulation in insomnia disorder. J Clin Sleep Med 2018;14:759-66.

40. Jiang H, Tian S, Bi K, et al. Hyperactive frontolimbic and frontocentral resting-state gamma connectivity in major depressive disorder. J Affect Disord 2019;257:74-82.

41. Yoshimura S, Okamoto $\mathrm{Y}$, Onoda $\mathrm{K}$, et al. Rostral anterior cingulate cortex activity mediates the relationship between the depressive symptoms and the medial prefrontal cortex activity. J Affect Disord 2010;122:76-85.

42. Lanius RA, Frewen PA, Vermetten E, et al. Fear conditioning and early life vulnerabilities: two distinct pathways of emotional dysregulation and brain dysfunction in PTSD. Eur J Psychotraumatol 2010: doi: 10.3402/ejpt.v1i0.5467.

43. Lee YG, Kim S, Kim N, et al. Changes in subcortical resting-state functional connectivity in patients with psychophysiological insomnia after cognitive-behavioral therapy: changes in resting-state FC after CBT for insomnia patients. Neuroimage Clin 2018;17:115-23.

44. Li M, Wang R, Zhao M, et al. Abnormalities of thalamus volume and resting state functional connectivity in primary insomnia patients. Brain Imaging Behav 2019;13:1193-201.

45. Andreasen NC, O'Leary DS, Cizadlo T, et al. Remembering the past: two facets of episodic memory explored with positron emission tomography. Am J Psychiatry 1995;152:1576-85.

46. Wang T, Yan J, Li S, et al. Increased insular connectivity with emotional regions in primary insomnia patients: a resting-state fMRI study. Eur Radiol 2017;27:3703-9.

47. Li X, Guo S, Wang C, et al. Increased interhemispheric restingstate functional connectivity in healthy participants with insomnia symptoms: A randomized clinical consort study. Medicine (Baltimore) 2017;96:e7037.

48. Liu CH, Liu CZ, Zhang J, et al., Reduced spontaneous neuronal activity in the insular cortex and thalamus in healthy adults with insomnia symptoms. Brain Res 2016;1648(Pt A):317-24.

49. Vantomme G, Osorio-Forero A, Luthi A, et al. Regulation of Local Sleep by the Thalamic Reticular Nucleus. Front Neurosci 2019; 13:576.

50. Crabtree JW, Isaac JT. New intrathalamic pathways allowing modalityrelated and cross-modality switching in the dorsal thalamus. J Neurosci 2002;22:8754-61.

51. Aron AR, Schlaghecken F, Fletcher PC, et al. Inhibition of subliminally primed responses is mediated by the caudate and thalamus: evidence from functional MRI and Huntington's disease. Brain 2003;126:713-23. 